\section{Quasar 4C39.25 is not contracting}

\author{
J. M. Marcaide*, N. Bartel $\dagger$, M. V. Gorenstein†, \\ I. I. Shapiroł, B. E. Coreył, A. E. E. Rogersł, \\ J. C. Webberł⿱亠乂, T. A. Clark§, J. D. Romney\| \\ \& R. A. Prestony
}

* Max-Planck-Institut für Radioastronomie, Auf dem Hügel 69 , D-5300 Bonn 1, FRG

† Harvard-Smithsonian Center for Astrophysics, 60 Garden St, Cambridge, Massachusetts 02138, USA

¥ Haystack Observatory, Westford, Massachusetts 01886, USA

$\S$ Goddard Space Flight Center, Greenbelt, Maryland 20771, USA

|| National Radio Astronomy Observatory, Edgemont Road, Charlottesville, Virginia 22901, USA

I Jet Propulsion Laboratory, 4800 Oak Grove Dr., Pasadena, California 91109, USA

The milli-arc second radio structure of the quasar 4 C39.25 has previously been described as consisting of two components whose angular separation remained constant at $\sim 2$ marc s, whereas their relative flux densities varied with time ${ }^{1-3}$. This behaviour is in marked contrast to other similar sources whose radio structures expand superluminally ${ }^{4}$. Recently, Shafier ${ }^{5}$ suggested that 4C39. 25 may have been contracting superluminally in the period 197982. Here, based on our map of this source made from VLBI observations in 1983 at $\lambda 3.6 \mathrm{~cm}$, we conclude that this conjecture is not correct. We find three distinct components in the structure, two of which are separated by $\mathbf{2 . 0}$ marc $\mathrm{s}$, whereas the third, presumably new and not previously reported, is situated between the other two. It is possible either that the third component is stationary and that its flux density has rapidly increased to render it visible, or that it has recently been ejected from the westernmost component.

Our VLBI observations of $4 C 39.25$ at a radio wavelength of $\lambda 3.6 \mathrm{~cm}$ took place on $10 \mathrm{May} 1983$. We recorded data for $2 \mathrm{~min}$ at $30 \mathrm{~min}$ intervals for over $7 \mathrm{~h}$. We used Mark III instrumentation $^{6}$, a synthesized bandwidth of $28 \mathrm{MHz}$, right-hand circular polarization and the following array of eight radio telescopes: $100 \mathrm{~m}$, at Effelsberg, FRG; $64 \mathrm{~m}$, at Robledo, Spain; $20 \mathrm{~m}$, at Onsala, Sweden; $37 \mathrm{~m}$, at Westford, Massachusetts, USA; $43 \mathrm{~m}$, at Green Bank, West Virginia, USA; $25 \mathrm{~m}$, at Fort Davis, Texas, USA; $64 \mathrm{~m}$, at Goldstone, California, USA; and $40 \mathrm{~m}$, at Big Pine, California, USA. Hydrogen maser frequency standards were used at each site. The data were processed at the Mark III processor of the Max-Planck-Institut für Radioastronomie in Bonn, FRG. After calibration of the visibility amplitudes ${ }^{7}$ we produced a map of the source using standard hybrid mapping techniques. To improve the relative calibration between the antennas we used the Cornwell and Wilkinson ${ }^{8}$ algorithm of self-calibration, which, given the large number of antennas available, worked very well. The resulting map is presented in Fig. 1.

We have labelled the components in Fig. 1 as $a, b$ and $c$. The separation of the centre of component $c$ from that of $a$ is $2.0 \pm 0.1 \mathrm{marcs}$; the separation of $b$ from $a$ is $1.4 \pm 0.1$ marc s. During the 1970 s the structure was well characterized at $\lambda 2$, $\lambda 2.8, \lambda 3.6$ and $\lambda 6 \mathrm{~cm}$ by two components separated by 2 marc $\mathrm{s}^{1-3}$. More recently, Shaffer ${ }^{5}$ mapped the source from observations at $\lambda 2.8$ and $\lambda 3.6 \mathrm{~cm}$ and found it to consist of two components, their angular separation significantly $<2.0$ marc $\mathrm{s}$, the western component being elongated. As a result, he conjectured that the source may be contracting, but he also recognized that motion in a source could be mimicked by the appearance of a new component. From our result we infer that the elongation of Shaffer's western component resulted from a blending of our two components $b$ and $c$, and conclude that our $a$ and $c$ com-

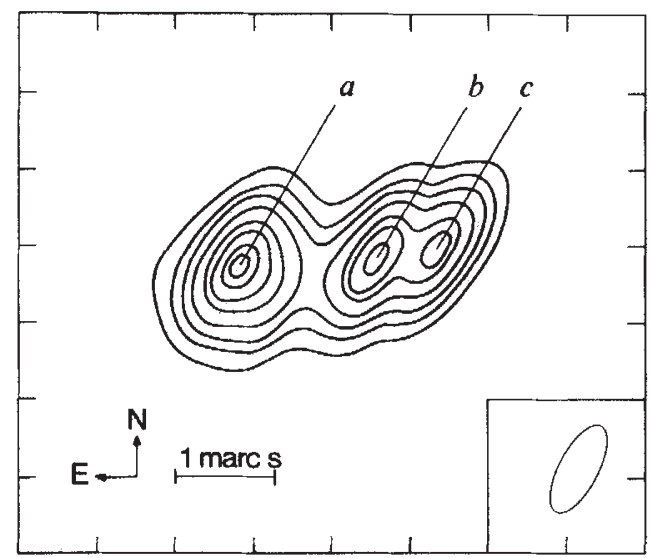

Fig. 1 Hybrid map of $4 \mathrm{C} 39.25$ made from $\lambda 3.6-\mathrm{cm}$ data from epoch 10 May 1983. The restoring beam used is shown boxed in the lower right-hand-side corner. The contours specified are 4, 10,

$18,30,50,65,85$ and $95 \%$ of the peak brightness temperature.

ponents correspond to those observed in the $1970 \mathrm{~s}$, that $b$ is a new component and that the source has, therefore, not contracted.

The origin of component $b$ is not clear: it could be a stationary component whose fiux density has rapidly increased or it may have been ejected, say, by component $c$. Curiously, according to H. D. Aller and M. F. Aller (personal communication) the flux density of $4 C 39.25$ has been decreasing steadily at wavelengths from $\lambda 2$ to $\lambda 6 \mathrm{~cm}$ since 1980 with no obvious new outbursts, whereas the linear polarization at $\lambda 2 \mathrm{~cm}$ has increased from 1 to $2.5 \%$ since 1983 . We cannot distinguish between the two proposed alternatives without the detailed analysis of other VLBI observations of comparable resolution made at different epochs. A detailed comparison of the results from our May 1983 observations, with observations at $\lambda 2.8 \mathrm{~cm}$ from June 1982 with similar resolution (which confirm the existence of the new $b$ component) and with observations at $\lambda 6 \mathrm{~cm}$ from July 1982, will be presented elsewhere.

If the component $b$ is stationary, $4 C 39.25$ would surprise us again by exhibiting a milli-arc second morphology quite different from that of other radio sources whose radio spectra and variability characteristics are shared by $4 \mathrm{C} 39.25$. On the other hand, if component $b$ has been ejected from component $c$, then its apparent motion may well be found to be superluminal. Moreover, it may overtake component $a$, as Shaffer ${ }^{5}$ noted. Such a possibility would be rather exciting, offering a unique opportunity to test different hypotheses on the nature of the components of compact radio sources.

We thank Mr. H. Blaschke for his assistance on the Mark III processor. J.M.M.'s research at the Max-Planck-Institut für Radioastronomie was supported in part by NASA-JPL Contract 956542. The Center for Astrophysics research was supported in part by the NSF.

Received 24 December 1984; accepted 3 February 1985. 